\title{
Self-assessment questions
}

\section{A young man with bronchial asthma and an abnormal chest X-ray}

\author{
A S Kashyap, S Kashyap
}

A 29-year-old man with bronchial asthma of 5 years duration was using inhaled salbutamol. In view of recurrent exacerbations, he had been put on oral prednisolone $20 \mathrm{mg} /$ day for the last year. He did not smoke tobacco or drink alcohol. He had no other complaints. Clinically he had moon facies, buffalo hump, centripetal obesity, purple striae on flanks and proximal myopathy. His blood pressure was $140 / 100 \mathrm{mmHg}$. Chest examination revealed polyphonic rhonchi in all areas. The rest of the general and physical examination was normal. Investigations revealed a normal haemogram, urinalysis, fasting and post-prandial plasma glucose, serum sodium, potassium, calcium and phosphate levels. Pulmonary function test showed an obstructive pattern. His chest $\mathrm{X}$-ray (postero-anterior) is shown in figure 1. Chest X-ray a year earlier had been normal. Serum cortisol levels were $130 \mathrm{nmol} / 1$ at $08.00 \mathrm{~h}$ (normal $140-690 \mathrm{nmol} / \mathrm{l}$ ) and $76 \mathrm{nmol} / 1$ at $16.00 \mathrm{~h}$ (80-330 nmol/l). Urine 24-hour calcium was $3.2 \mathrm{mmol}(<3.8 \mathrm{mmol})$.

\section{Department of \\ Medicine, Armed \\ Forces Medical \\ College, Pune 411040, \\ India \\ A S Kashyap \\ Cardiothoracic \\ Centre, Golibar \\ Maidan, Pune 411040, \\ India \\ S Kashyap}

Submitted 29 March 1999 Accepted 13 May 1999

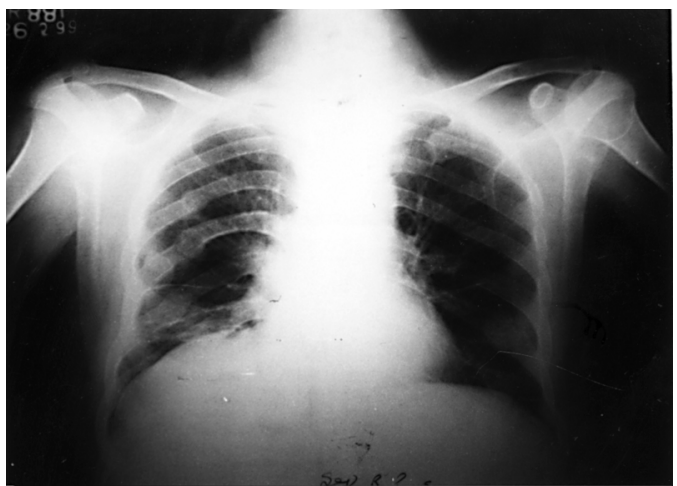

\section{Questions}

1 What are the abnormalities seen on the chest $\mathrm{X}$-ray?

2 What is the pathophysiology of these abnormalities?

Figure Chest X-ray (postero-anterior) 
Answers

QUESTION 1

The chest X-ray reveals diffuse osteoporosis of the ribs and bilateral multiple rib fractures with partial healing and exuberant calcified callus (pseudocallus) surrounding a radiolucent zone of nonunion in this patient with iatrogenic Cushing's syndrome. Abundant pseudocallus is a hallmark of corticosteroid-induced osteoporosis. It is seen most frequently around stress fractures in the ribs, pelvis and end plates of collapsed vertebrae. Microscopically, there is a reduction in osteoblastic activity and production of a cartilaginous callus that becomes highly mineralized in an amorphous fashion. Spontaneous symptomless fractures are typically seen in Cushing's syndrome (endogenous and exogenous). In addition to the ribs, these fractures may also occur in feet, vertebrae, pubic and ischial rami, and uncommonly in long bones. They may superficially resemble pseudofractures of osteomalacia. At times these fractures may be the presenting sign of Cushing's syndrome, particularly in men. ${ }^{2}$

The estimated incidence of fractures in patients prescribed corticosteroids is $30-50 \%{ }^{3}$ Bone loss occurs rapidly within first 6-12 months after therapy is begun, rates slowing down subsequently. Rates of bone loss are directly related to corticosteroid dose; significant trabecular bone loss occurs with prednisone doses greater than $7.5 \mathrm{mg} /$ day. ${ }^{4}$ Bone loss occurs even with inhaled steroids.

Bone loss is greater at trabecular than at cortical sites. Fractures are predominant at sites rich in trabecular bones such as vertebral bodies and ribs, but risk of hip fracture is also tripled due to bone loss from proximal femur, particularly Ward's triangle, because it is composed of trabecular bone. ${ }^{4}$ Over a period of time, steroids affect cortical bones and fragility of long bones is increased.

In corticosteroid-induced osteoporosis, vertical and horizontal trabeculae are equally thin and lead to a uniformly translucent appearance of the vertebrae, whereas in postmenopausal osteoporosis, horizontal trabeculae are predominantly lost, and lead to a 'corduroy stripe' appearance.

The usual risk factors for osteoporosis do not apply to same extent to glucocorticoid-induced bone loss. Young adult men receiving glucocorticoids lose bone more rapidly than do older men, postmenopausal and premenopausal women. However, postmenopausal women receiving equivalent doses of steroids are at greater risk for fracture, presumably because they have a lower bone mass when they initiate steroid therapy. Patients with rheumatoid arthritis, chronic pulmonary and gastrointestinal diseases are at increased risk because disease associated inflammation, poor nutrition and immobilisation can aggravate bone loss. Patients who undergo organ transplant are at particular risk. Severe bone loss due to steroids may occur without other side effects, though there is a strong association between glucocorticoid-induced myopathy and osteoporosis. $^{5}$
Skeletal manifestations of Cushing's syndrome

- osteoporosis, predominantly of trabecular bones

- spontaneous symptomless fractures of ribs, pubic and ischial rami, feet bones and vertebrae

- healing of fractures with abundant pseudocallus formation

- osteonecrosis (avascular necrosis) of head of femur and humerus, distal femur and vertebrae

- suppression of growth velocity in children

QUESTION 2

The pathophysiology of osteoporosis due to corticosteroids is a relative increase in bone resorption and decrease in bone formation. Reduced bone formation is due to direct inhibitory effects of corticosteroids at supraphysiological doses on osteoblast numbers, lifespan and function, leading to inhibition of synthesis of bone collagen by pre-existing osteoblasts and diminished conversion of precursor cells to functioning osteoblasts. Glucocorticoids also inhibit the synthesis of other bone proteins, including osteocalcin, a major bone matrix protein. Recent studies have found that glucocorticoids accelerate the apoptosis of osteoblast cells. ${ }^{6}$

Pharmacological doses of glucocorticoids inhibit synthesis or the actions of growth factors with anabolic effects on bone. This includes growth hormone, insulin-like growth factor 1 (IGF-1) and transforming growth factor $\beta$ (TGF- $\beta)$. Glucocorticoids may inhibit bone formation by prostaglandin synthesis inhibition.

Increased osteoclast recruitment and associated parameters of bone resorption, including eroded surfaces and calcium kinetics, may also be increased. Osteoclastic effects may be mediated by secondary hyperparathyroidism. A combination of corticosteroid-induced increased urinary calcium excretion and reduced intestinal absorption may be responsible for secondary hyperparathyroidism. Corticosteroids do not cause significant abnormalities in vitamin $\mathrm{D}$ metabolism.

Corticosteroids alter gonadal function, which may indirectly influence mineral metabolism by increased bone resorption. A combination of inhibition of pituitary gonadotropin secretion and direct effects on the ovary or testes, lead to a reduction in production of oestrogen and testosterone. Glucocorticoids blunt the secretion of luteinising hormone in response to luteinising hormone-releasing hormone in both men and women. ${ }^{8}$ They inhibit follicle-stimulating-hormone-induced oestrogen production, and decrease testosterone production. Circulating levels of androstenedione and oestrone are suppressed further by reduced adrenal production of androstenedione, caused by the suppression of adrenocorticotropin due to corticosteroid therapy, and by the resultant adrenal atrophy. Oestrogen deficiency and corticosteroids may have an additive effect in increasing the rate of bone loss. ${ }^{9}$ 


\section{Mechanisms of glucocorticoid osteoporosis}

- direct suppressive effect on osteoblasts

- decreased gut absorption of calcium

- increased urinary calcium excretion

- secondary hyperparathyroidism, leading to increased osteoclastic bone resorption

- sex hormone effects (diminished adrenal androgens, oestrogen and testosterone) leading to increased osteoclastic bone resorption

- catabolic effects on proteins, including muscle and bone matrix

- diminished synthesis and/or action of growth hormone, IGF-1, TGF- $\beta$

In our patient, skeletal survey revealed diffuse osteoporosis, but no fractures in the vertebrae or elsewhere. He could not afford bone mineral density measurement. His treat-

1 Adachi JD. Corticosteroid induced osteoporosis. Am $\mathcal{F} \mathrm{Med}$ Sci 1997;313:41-9.

2 Urbanic RC, George JM. Cushing's disease-18 years' experience. Medicine 1981;60:14-24.

3 Ruegsegger P, Medici TC, Anliker M. Corticosteroid induced bone loss. A longitudinal study of alternate day therapy in patients with bronchial asthma using quantitative computed patients with bronchial asthma using quantitative com

4 Adachi JD, Bensen WG, Bell MJ, et al. Corticosteroid induced osteoporosis: follow-up over 3 years. In: Chrisinduced osteoporosis: follow-up over 3 years. In: ChrisInternational Symposium on Osteoporosis. Copenhagen, International Symposium on Osteoporosis.

5 Askari A, Vignos PJJ, Moskowitz RW. Steroid myopathy in connective tissue diseases. Am f Med 1976;61:485. ment of bronchial asthma was optimised with inhaled salbutamol and beclomethasone. Oral steroids were gradually tapered over the next 6 weeks and stopped. He was put on a sodiumrestricted diet $(3 \mathrm{~g} / \mathrm{d})$, elemental calcium 1500 $\mathrm{mg} / \mathrm{d}$, vitamin D3 $800 \mathrm{IU}$ and alendronate 10 $\mathrm{mg} / \mathrm{d}$. He was started on a weight bearing and isometric exercise programme. Over the last 6 months there has been no exacerbation of asthma and rib fractures have shown partial healing.

\section{Final diagnosis}

Corticosteroid-induced osteoporosis in a patient with Cushing's syndrome.

Keywords: corticosteroids; osteoporosis; Cushing's syndrome

6 Weinstein RS, Jilka RL, Miller FL, et al. Glucocorticoid excess causes apoptosis of osteocytes in murine cortical bone: a potential explanation for bone necrosis. F Bone Miner Res 1997; (suppl 1):S142.

7 Reid IR. Preventing glucocorticoid-induced osteoporosis. $N$ Engl f Med 1997;337:420-1.

8 Luton JP, Thiebolt P, Valcke JC, et al. Reversible gonadotropin deficiency in male Cushing's disease. 7 Clin Endocrino Metab 1977;45:488-95.

9 Goulding A, Gold E. Effects of chronic prednisolone treatment on bone resorption and bone composition in intact and ovariectomized rats receiving B-estradiol. Endocrinology 1988;122:482-7

\section{A rare cause of right-sided abdominal pain in a young woman}

Level 5, Belfast City Hospital, Lisburn Road, Belfast BT9

$7 \mathrm{AB}, \mathbf{N}$ Ireland W K Edrees

South Tyrone Hospital, Carland Road,

Dungannon BT71 4AU, Co Tyrone, $N$ Ireland M Hussien

Downe Hospital, Downpatrick

BT30 6JA, Co Downe, N Ireland

M Ismail

Submitted 23 April 1999 Accepted 3 June 1999

\author{
W K Edrees, M Hussien, M Ismail
}

An 18-year-old woman gravida 0 , para 0 , was admitted with a 3-week history of vague right-sided abdominal pain. She denied any gastrointestinal or urinary symptoms or vaginal discharge. Her last menstrual period was one week prior to admission and was normal. She was sexually active. Her medical history was unremarkable. On examination, she was apyrexic with tenderness along the right side of the abdomen extending from the costal margin to the right iliac fossa, with no guarding or rebound tenderness. Bowel sounds were normal. Pelvic examination revealed slight tenderness on the right side with no masses. White cell count was $5.5 \times 10^{9} / 1$, erythrocyte sedimentation rate was not elevated. Pregnancy test and MSSU were negative. Chest and abdominal $\mathrm{X}$-ray was normal. Ultrasound scan showed a small amount of fluid in the pelvis with normal ovaries. She was managed conservatively, but her abdominal pain and tenderness persisted.

\section{Questions}

1 What other investigation would you consider?

2 What is the probable diagnosis? 
Answers

QUESTION 1

Diagnostic laparoscopy is indicated here in view of atypical pain and non-characteristic signs with non-diagnostic ultrasound scan findings. It showed a normal appendix, inflamed right fallopian tube with serosanguineous fluid in the pelvis. The anterior surface of the liver was inflamed and covered with haemorrhagic exudate.

QUESTION 2

This is a case of perihepatitis associated with pelvic inflammatory disease, known as FitzHugh-Curtis syndrome (FHC). The patient was treated with tetracycline and metronidazole. Her abdominal pain settled dramatically in 24 hours and she was discharged home in the third postoperative day.

\section{Discussion}

The cause of right-sided abdominal pain in a fertile woman is sometimes difficult to diagnose, especially if it is not associated with classical symptoms and signs. The association between pelvic inflammatory disease (PID) and perihepatitis was first described by Curtis in $1930,{ }^{1}$ followed by Fitz-Hugh in $1934 .^{2}$ The syndrome is more commonly observed nowadays, a phenomenon directly associated with the increasing incidence of PID. ${ }^{3}$

Initially, FHC syndrome was seen as a result of gonorrhoeal infection. Recently, Chlamydia trachomatis infection has been found to play a more significant role. ${ }^{4}$ In the acute stage petechial haemorrhages and fibrinous exudate are observed on the liver surface and pelvic organs. This leads to adhesions within the pelvic organs and between liver surface and surrounding organs (violin-string adhesions). ${ }^{5}$ Different mechanisms have been postulated for the extension of pelvic infection to the liver capsule, including transcoelmic spread via the right paracolic space, retroperitoneal lymphatic and haematogenous spread. ${ }^{6}$ It was suggested that chlamydial perihepatitis may be due to a hyperimmune reaction.?

1 Curtis AH. A cause of adhesions in the right upper quadrant. $7 A M A$ 1930;94:1221-2.

Fitz-Hugh T. Acute gonococcic peritonitis of the right upper quadrant in women. $\mathscr{F} A M A$ 1934;102:2094-6.

3 Shannon M. A young woman with pleuritic right upper quadrant pain. Hosp Pract 1984:June:171-3.

4 Wang S-P, Eschenbach DA, Holmes KK, Wager G Grayston JT. Chlamydia trachomatis infection in FitzHugh-Curtis syndrome. Am f Obstet Gynecol 1980;138: 1034-8.

5 Toshihiko T, Hiroshi H, Wai-IP, Akira Y. Fitz-Hugh-Curtis syndrome: three cases confirmed by laparoscopy. AsiaOceania f Obstet Gynecol 1990;16:105-10.

\section{Learning points}

- a high index of suspicion of FHC syndrome should be considered in cases of right-sided abdominal pain in sexually active young women

- laparoscopy is essential for the diagnosis of atypical abdominal pain and may reveal rare causes

The classic presenting symptom is pleuritic right upper abdominal pain with possibly bilateral upper abdominal pain and tenderness in the presence of acute or subacute PID. ${ }^{8}$ The clinical diagnosis can be difficult and other causes of right-sided abdominal pain have to be excluded. Cultures of the endocervix for $C$ trachomatis and Neisseria gonorrhea may be difficult and a negative culture does not exclude the diagnosis since the sensitivity of a single culture is limited.

Serologic evidence of acute chlamydial infection can be obtained by demonstrating the presence of $\operatorname{IgM}$ antibody, a rise in antibody titre in serial sera, or a very high titre of IgG antibody. ${ }^{4}$ Laparoscopy plays an essential role in the diagnosis of atypical abdominal pain. Perihepatitis cannot be diagnosed with certainty unless it is directly visualised. ${ }^{7}$

The findings in cases of FHC syndrome range from haemorrhagic appearance of the liver, to dense adhesions between the liver and surroundings associated with inflamed fallopian tubes. ${ }^{5}$ Antibiotic therapy specific for the possible causative organisms ( $C$ trachomatis, gonococci and anaerobes) gives a dramatic response; a combination of tetracycline (or doxycycline) and metronidazole provides excellent coverage. ${ }^{3}$

\section{Final diagnosis}

Perihepatitis associated with pelvic inflammatory disease (Fitz-Hugh-Curtis syndrome)

Keywords: adnexitis; hepatitis; abdominal pain; pelvic inflammatory disease; Fitz-Hugh-Curtis syndrome

6 Corson SL. The Fitz-Hugh-Curtis syndrome revisited: changing perspectives after half a century. $\mathcal{F}$ Reprod Med 1985;30:567-82.

7 Eschenbach DA, Wolner-hanssen P. Fitz-Hugh-Curtis syndrome. In: Holmes KK, Mardh PA, Sparling PF, Wiesner PJ, eds, Sexually transmitted diseases. New York: McGraw-Hill, 1990; pp 621-6.

McGraw-Hill, 1990; pp 621-6.
8 Fransen L, Avonts D, Piot P. Genital chlamydial infection associated with perihepatitis. Acta Clin Belg 1982;37:324-7. 


\title{
A lung cyst following blunt chest trauma
}

\author{
P Sundaram, K Agrawal, D Balakrishnan, R T Kamble, J M Joshi
}

A 48-year-old woman with no significant medical history was admitted following a road traffic accident. She developed right-sided pleuritic chest pain, a dry cough and severe breathlessness. On physical examination, the patient had a pulse rate of 90 beats $/ \mathrm{min}$, blood pressure of 130/80 $\mathrm{mmHg}$ and a respiratory rate of $30 \mathrm{breaths} / \mathrm{min}$. There was crepitus on the right chest wall and breath sounds were markedly diminished on that side. She also had severe tenderness in the region of her left shoulder. Cardiovascular, abdominal and neurological examination did not reveal any abnormality.

A chest X-ray on admission revealed rib fractures from the second to the sixth ribs, subcutaneous emphysema and a pneumothorax on the right side (figure 1). She had also sustained a fracture of the neck of the left humerus. Baseline blood investigations showed a haemoglobin of 11.6 $\mathrm{g} / \mathrm{dl}$, white blood count of $12.6 \times 10^{6} / 1$. Arterial blood gas analysis revealed moderate hypoxaemia. An intercostal tube drainage was instituted in the right thoracic cavity. Her subsequent chest $\mathrm{X}$-ray revealed a cystic lesion in the right lower lobe following complete expansion of the right lung (figure 2).

Department of Respiratory Medicine, Topiwala National Medical College, BYL Nair Ch Hospital,

Mumbai 400008, India

P Sundaram

K Agrawal

D Balakrishnan

R T Kamble

J M Joshi

Submitted 21 August 1998 Accepted 26 May 1999

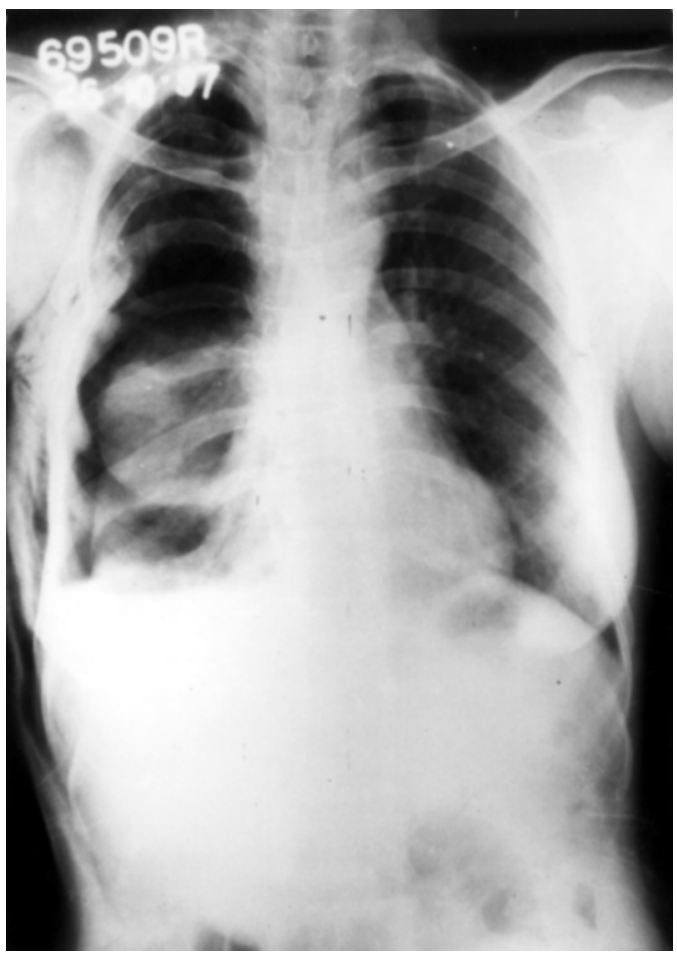

Figure 1 Chest X-ray showing rib fractures, subcutaneous emphysema and pneumothorax on the right side

\section{Questions}

1 What is the differential diagnosis?

2 What is the most probable diagnosis?

3 What is the treatment of this condition?

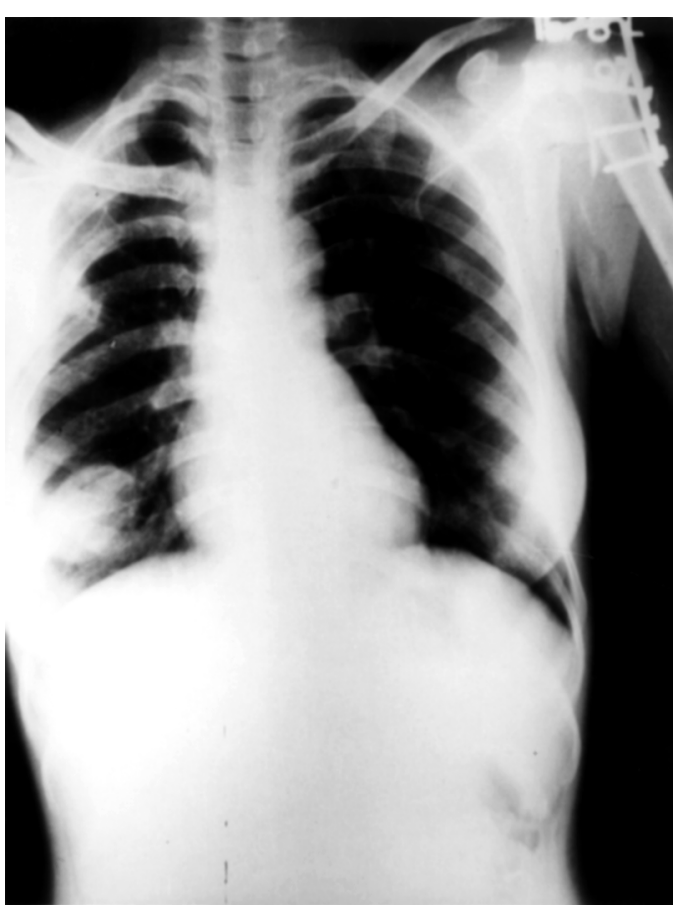

Figure 2 Right lower lobe cystic lesion seen on the chest X-ray 
Answers

QUESTION 1

The various aetiologies to be considered following trauma are pulmonary haematoma, contusion, traumatic lung cyst, loculated haemopneumothorax. ${ }^{1}$ Also to be considered in the differential diagnosis are pre-existing conditions in an adult like lung abscess, tuberculous and mycotic cavities, hydatid cysts, cavitating bronchogenic carcinomas and, in children, congenital pulmonary cyst, postpneumonic pneumatocoele and sequestration.

QUESTION 2

The most probable diagnosis is traumatic lung cyst.

QUESTION 3

"Clinical course of traumatic lung cysts is usually uncomplicated". Complete resolution occurs in 2-16 weeks requiring only close observation and symptomatic treatment. Prophylactic antibiotic treatment is not required, despite transient fever and leucocytosis. Secondary infection of traumatic lung cyst with abscess formation is uncommon. Very rarely it may fail to regress and may enlarge resulting in respiratory distress or pneumothorax.

When the patient was seen a month later following discharge, the cystic lesion had completely disappeared (figure 3 ).

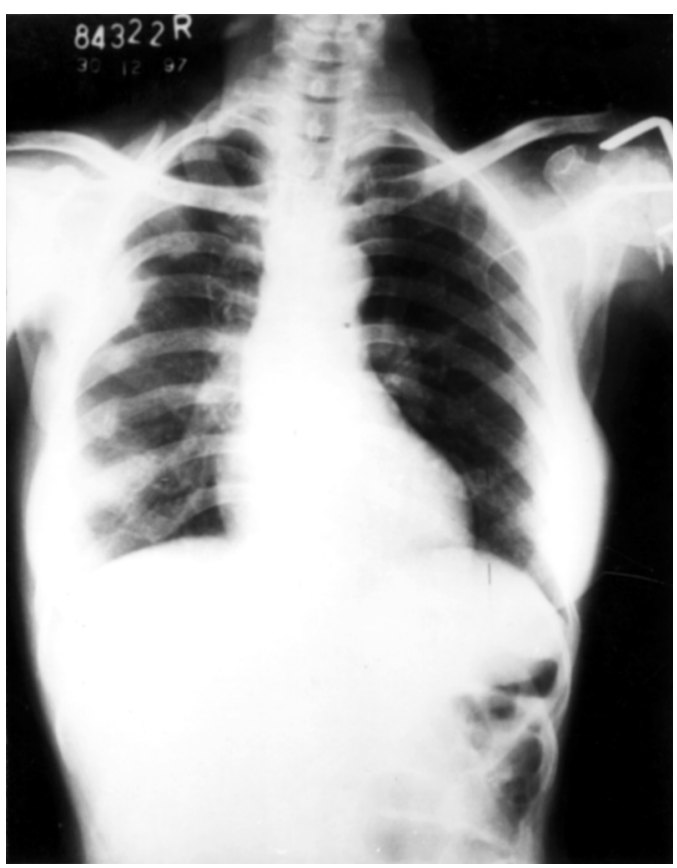

Figure 3 Chest X-ray a month after discharge

\section{Discussion}

Lung laceration due to trauma may result in pneumothorax, haemothorax, contusion, traumatic pseudocyst and massive haemoptysis. ${ }^{2}$ Blunt chest trauma causes contusion while penetrating injury gives rise to haematoma.

\section{Traumatic lung cysts}

- may occur following blunt chest trauma

- usually appear within 12-24 hours of injury

- may be single or multilocular, ranging from 2-14 $\mathrm{cm}$

- spontaneous regression occurs in 2-6 weeks

- it is important to recognise such lesions to avoid confusion with other causes of lung masses or cavities

Recognition of traumatic lung cysts is important to avoid confusion with other causes of lung masses or cavities as it resolves spontaneously requiring no special treatment.

Closed chest trauma may result in the development of one or more cystic spaces within the lung that may remain airfilled or may partly fill. The trauma is usually blunt. Children and young adults seem to be more prone because of greater flexibility of their thoracic walls. In a recent review of 40 cases of traumatic lung cyst, $88 \%$ occurred in patients under 32 years of age. Three mechanisms have been suggested to explain their development ${ }^{13}$ :

- sudden compression of an area of the lung closes off a segment of peripheral bronchial tree and creates within it a bursting, explosive, pressure that is expended in the rupture of successive alveolar walls within the lobules supplied by the occluded bronchus

- compression of the elastic chest wall while the glottis is closed may result in rupture of the small bronchi and pulmonary parenchymal disruption with cavitation because air from the compressed part of the lung cannot escape quickly enough

- a concussion wave produced by the blow to the chest generates shearing stress that tears the lung parenchyma and causes traumatic cavities.

The cyst may contain air or blood derived from the torn alveolar capillaries. The cyst wall is thin and is made up of fibrous tissue and surrounding compressed alveolar tissue. Rarely, there may be colonisation of a posttraumatic lung cyst by an asymptomatic aspergilloma. ${ }^{4}$

Symptoms include haemoptysis, chest pain, cough, dyspnoea and low grade fever. Haemoptysis is present in $50 \%$ of cases and may last for several days. Low grade fever and leucocytosis lasting for one to two weeks reflect reparative process of the injured lung.

The findings on chest X-ray depend on timing after the injury, associated pulmonary parenchymal injuries (contusion/haematoma), and whether the cyst is filled with air and/or blood. ${ }^{1-35}$ The cyst may be single or multiple, unilocular or multilocular ranging from 2-14 $\mathrm{cm}$, located subpleurally and usually appears 12-24 hours after injury. Occasionally there may be a delayed appearance 3-6 days after the resolution of the surrounding lung contusion. In comparison, lung contusions usually appear 
within one hour, resolve in $48-72$ hours, and disappear in 7-10 days.

Spontaneous regression occurs in 2-6 weeks although the cyst may occasionally persist for up to 4 months. Surgical resection is not necessary provided there is definite evidence of decreasing size of the lesion by 6 weeks following injury in adults and by 3 months in children. Surgery is indicated if there is an

1 Shamji FM, Sachs HJ, Perkins DG. Cystic disease of the lungs. Surg Clin N Am 1988;68:581-620.

2 Peter Pare JA, Fraser RG. Synopsis of the disease of the chest. In: ? eds, Diseases of the thorax caused by external physical agents. Philadelphia: WB Saunders, 1983; pp 616-39.

3 Guenter CA. Chest trauma. In: Pulmonary medicine, Guenter CA. Chest trauma. In: Pulmonary medicine,
Philadelphia: JB Lippincott Company, 1982; pp 512-54. infective complication unresponsive to antibiotic treatment or if other cavitatory lesions of the lung are to be excluded.

\section{Final diagnosis}

Traumatic lung cyst.

Keywords: chest trauma; lung cyst; trauma

4 Torre W, Dominguez L. The colonisation of post traumatic lung cyst by an asymptomatic aspergilloma. Ann Intern Med 1997; 14:539.

5 Shingawa S, Fujimura M, Mizuhashik M, et al. Traumatic lung cyst. Respir Med 1996;90:115-6.

\title{
Neurological deterioration in a patient with myeloma
}

\author{
A J Logan, J E Grey
}

A 58-year-old woman with previously asymptomatic multiple myeloma presented acutely to the emergency medical intake with a severe frontal and left-sided headache. On examination she was apyrexial and normotensive and the rest of the general and, in particular, the neurological examination was normal. Investigations at that time revealed a haemoglobin of $8.8 \mathrm{~g} / \mathrm{dl}$, white cell count of $5.01 \times 10^{9} / 1$, erythrocyte sedimentation rate of $138 \mathrm{~mm} / \mathrm{h}$ and plasma viscosity of 3.6 centipoise (1.5-1.72). Serum electrophoresis revealed a paraprotein level of $25 \mathrm{~g} / 1$ (IgA $)$, a $\kappa: \lambda$ ratio of 16.2 (1-2.7) and a $\beta_{2}$-microglobulin of $2.2 \mathrm{mg} / 1$ (1.2-2.4). Bone marrow aspirate demonstrated $18 \%$ plasma cells (normal $<5 \%$ ). Twelve hours post admission she developed neurological symptoms and orbital pain. An urgent computed tomography (CT) scan of the orbits and paranasal sinuses was performed (figure). The lesion demonstrated on the CT was removed surgically.

\section{Department of Integrated Medicine, University Hospital of Wales, Heath Park, Cardiff CF4 4XW UK A J Logan J E Grey}

Submitted 29 March 1999 Accepted 3 June 1999

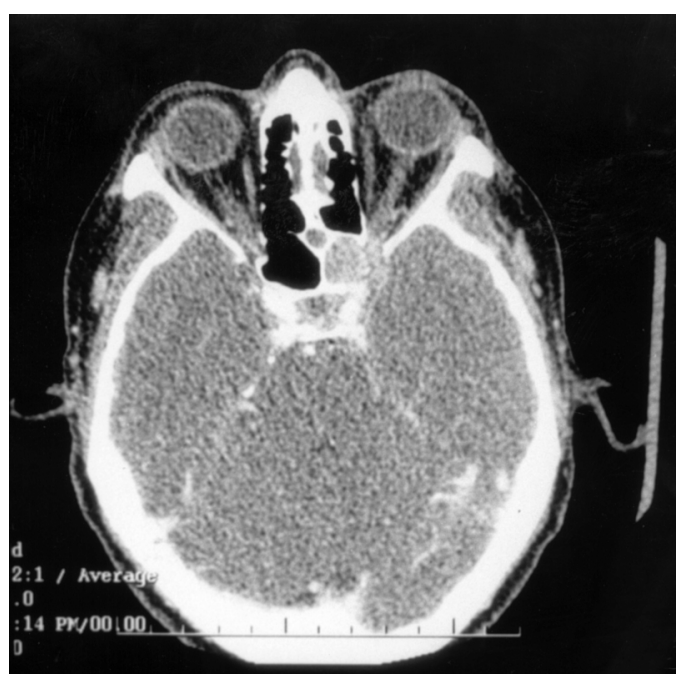

\section{Questions}

1 What is the probable diagnosis and differential diagnosis?

2 Where are these tumours most commonly found?

3 Given the site of the lesion, what neurological signs might have accompanied her deterioration?

4 What would be the treatment of choice for a solitary tumour such as this?
Figure CT scan of orbits (reproduced with the permission of the patient's next of kin) 


\section{Answers}

QUESTION 1

The diagnosis is a left retro-orbital plasmacytoma. Differential diagnosis is shown in box 1 .

\section{Differential diagnosis}

Nasal passage and sinus tumours:

- benign: haemangioma, squamous papilloma

- malignant: squamous cell carcinoma, adenocarcinoma

Craniopharyngioma

Box 1

A plasmacytoma is a not uncommon complication of multiple myeloma. It may rarely manifest as a primary tumour, occurring without evidence of other disease. In our patient histology of the removed mass demonstrated mitotic figures in a plasmacytoid tumour cell infiltrate. Immunostaining demonstrated positivity for IgG, IgA, and $\kappa$-light chain consistent with myeloma, suggesting spread of preexisting disease.

QUESTION 2

Extramedullary plasmacytomas (EMP) may involve any extramedullary organ or tissue but most are found in the head and neck region, usually in the mucosa of the oronasopharynx or paranasal sinuses. ${ }^{1}$ It is, however, rare for this solitary tumour to involve the orbit. ${ }^{2}$ EMPs account for approximately 3\% of all paranasal sinus and nasal cavity tumours. ${ }^{3}$ Localised pain and epistaxis are the two most common presentations with proptosis and sixth nerve palsy being much rarer.

1 Berbsagel DE, Pruzanski W. Syndromes and special presentations associated with multiple myeloma. In: Wiernik PH, Canellos GP, Kyle RA, Schiffer CA, eds. Neoplastic diseases of the blood, 3rd edn. Churchill Livingstone, 1996; pp 585-600. the blood, 3rd edn.Churchill Livingstone, 1996; pp 585-600. Mewis-Levin L, Garcia C, Olson S. Plasma
the orbit. Ann Ophthalmol 1981;17:447-81.

the orbit. Ann Ophthalmol 1981;17:447-81.
Castro E, Lewis J, Strong H. Plasmacytoma of the paranasal sinuses and nasal cavity. Arch Otolaryngol 1973;97:326-9.

\section{Learning points}

- EMP may complicate multiple myeloma

- EMP is most often found in the head and neck especially in the oronasopharynx and paranasal sinuses

- localised pain and epistaxis may be the presenting features of orbital/paranasal EMP

- EMP may threaten sight

- there should be a low threshold of suspicion of an EMP in patients with myeloma who develop neurological symptoms

- the treatment of choice of an EMP is radiotherapy

Box 2

QUESTION 3

The rapid progression of the neurological symptoms was related to the mass effect of the tumour, possibly secondary to haemorrhage into the lesion. As the lesion was located at the apex of the orbit this led to:

- diplopia secondary to a left sixth and partial third nerve palsy

- a left relative afferent pupillary defect

- rapid deterioration in vision in the left eye.

\section{QUESTION 4}

EMPs are highly radiosensitive and radiotherapy is potentially curative for a primary EMP. Surgery is reserved as a second-line treatment according to the tumour's response to radiotherapy. ${ }^{5}$ However, in patients with pre-existing multiple myeloma, radiotherapy and surgery are used more often as palliative procedures, in addition to the treatment of the underlying condition.

\section{Final diagnosis}

Left retro-orbital plasmacytoma.

Keywords: plasmacytoma; myeloma; head and neck tumours

Kapadia S, Desai U, Cheng V. Extramedullary plasmacytoma of the head and neck. A clinicopathologic study of 20 cases. Medicine 1982;51:317-29.

5 Nofsinger YC, Mirza N, Rowan PT, Lanza D, Wienstein G. Head and neck manifestations of plasma cell neoplasms. Laryngoscope 1997;107:741-6. 


\title{
A 30-year-old man with stroke and skin lesions
}

\author{
K N Viswanathan, S Anandan, S Sreenivas, K S Kumar, S Raman
}

30-year-old man was admitted to our hospital with a depressed conscious level (Glasgow Coma Scale score 4/15), following an attack of generalised tonic-clonic convulsions. He had smoked 30 cigarettes a day for 5 years prior to the fit, but had not consumed alcohol. There was no history of hypertension, diabetes mellitus, jaundice, fever, bleeding diatheses, trauma or prolonged bed rest.

General examination revealed a man who was apyrexial with normal pulse rate and blood pressure and livedo reticularis of the lower limbs (figure 1). Neurological examination revealed normal sized pupils, equal and sluggishly reacting to light. Oculocephalic reflexes were absent. There was no neck stiffness or lateralising limb weakness, plantars being bilaterally extensor. Cardiovascular, respiratory and abdominal examinations were clinically normal and a detailed search for malignancy proved negative.

Cranial computed tomography (CT) showed thrombosis of the straight and superior sagittal sinuses, subsequently confirmed by magnetic resonance imaging and angiography (MRI/MRA) (figure 2). Patient had thrombocytopenia $\left(90 \times 10^{9} / 1\right)$, normal erythrocyte sedimentation rate and a prolonged partial thromboplastin time (PTT). Liver function tests, whole and red blood cell counts, haemoglobin, bleeding and clotting times were normal.

He was subjected to further special investigations which clinched the diagnosis. Incidentally, the brother and sister of the patient were found to have leg ulcers and the biochemical abnormalities which the patient had on special investigations.

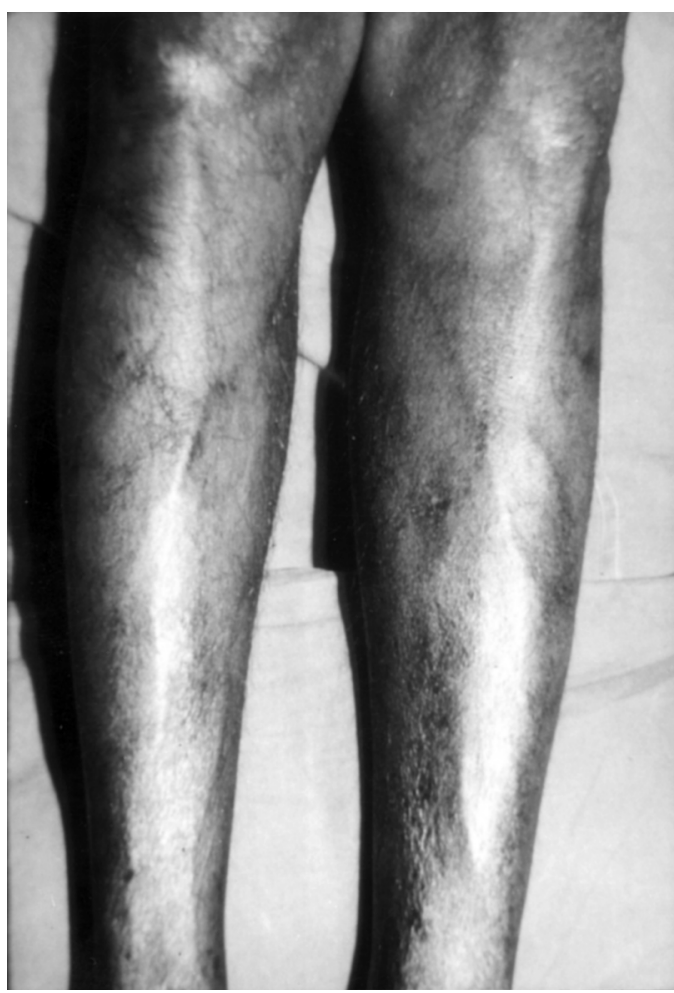

Figure 1 Livedo reticularis of the lower limbs

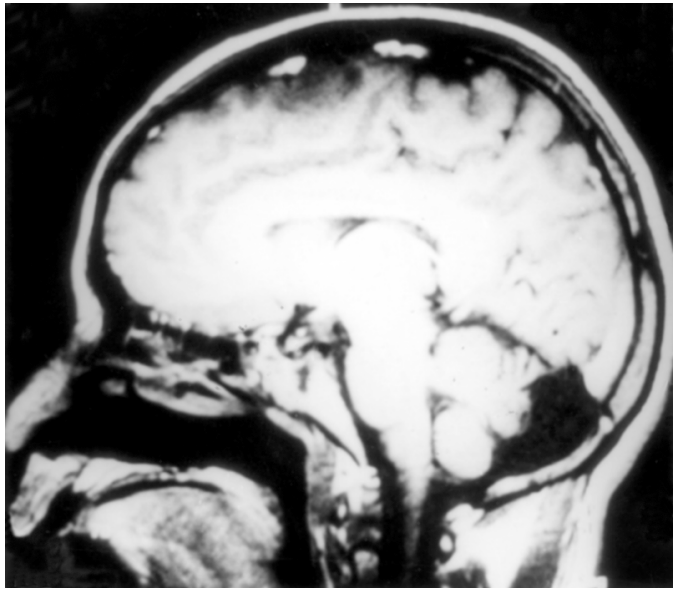

Figure 2 Admission MRI

S Anandan

S Sreenivas

K S Kumar

S Raman

Correspondence to

Dr K N Viswanathan, 6 First Cross Street, Sylvan Lodge Colony, Kilpauk,

Chennai-600010, India

Submitted 9 June 1998 Accepted 26 May 1999

1 What is the most likely diagnosis?

2 What further investigations would establish the aetiology of cerebral venous thrombosis?

3 What is the line of management to be pursued?

4 What is the significance of the skin lesions? 
Answers

QUESTION 1

The cerebral venous thrombosis could be the result of an underlying hypercoagulable state. The thrombocytopenia and prolonged PTT may point to the diagnosis of antiphospholipid syndrome (APLS).

QUESTION 2

APLS was confirmed in this patient by estimation of antiphospholipid antibodies in serum (anticardiolipin antibody and lupus anticoagulant). IgG and IgM anticardiolipin antibodies were markedly elevated ( $>5 \mathrm{SD}$ ) and lupus anticoagulant was positive (prolonged PTT with dilute thromboplastin). Other methods of estimation of lupus anticoagulant are by Russel's viper venom time and kaolin clotting time. These antibodies are known to be present in systemic lupus erythematosus. However, antinuclear antibodies, and antibodies to double-stranded DNA and lupus cells were found to be negative, making the possibility of systemic lupus erythematosus highly unlikely. Relevant investigations to rule out other hypercoagulable states (protein $\mathrm{C}$, protein $\mathrm{S}$ and antithrombin-III levels) were also normal. Urinary cyanide nitroprusside test for homocystinuria was negative, as was a sucrose lysis test for paroxysmal nocturnal haemoglobinuria.

Serum electrophoresis showed normal protein bands and blood for sickling, malarial parasite, leptospiral antibody titre, HIV, HbsAg and VDRL were negative. There was no evidence of DIC (normal serum fibrinogen, thrombin time and fibrin degradation products). Total red cell count and packed cell volume were normal, ruling out polycythaemia. Peripheral smear did not reveal abnormal cells. An echocardiogram showed neither clots in the cardiac chambers nor vegetations.

QUESTION 3

Anticerebral oedema measures, intravenous gamma globulin and plasmapheresis have a role in acute catastrophic APLS. Anticoagulants including aspirin are the mainstay of treatment, maintaining an INR of $>3$, and steroids are also recommended.

Our patient was treated initially with anticerebral oedema measures, aspirin, heparin and methylprednisolone, with good supportive care, switching over to warfarin, aspirin and prednisolone after 3 weeks. He is now on warfarin, aspirin and low-dose prednisolone. The anticardiolipin antibodies are positive in low titres $(<2 \mathrm{SD})$, and lupus anticoagulant is still positive.

QUESTION 4

Livedo reticularis has been reported to be associated with cerebrovascular accidents and antiphospholipid antibodies (Sneddon's syndrome).

\section{Clinical features of antiphospholipid} syndrome

- arterial and venous thromboses

- thrombocytopenia

- chorea

- haemolytic anaemia

- livedo reticularis

- leg ulcers

- heart valve disease

- recurrent spontaneous abortions

\section{Outcome}

After hospital treatment for about 6 weeks, he had fully recovered except for minimal bilateral sensorineural deafness (a consequence of the sagittal sinus thrombosis), detected on an audiogram. A repeat MRI showed recanalisation of the thrombosed sinuses (figure 3 ).

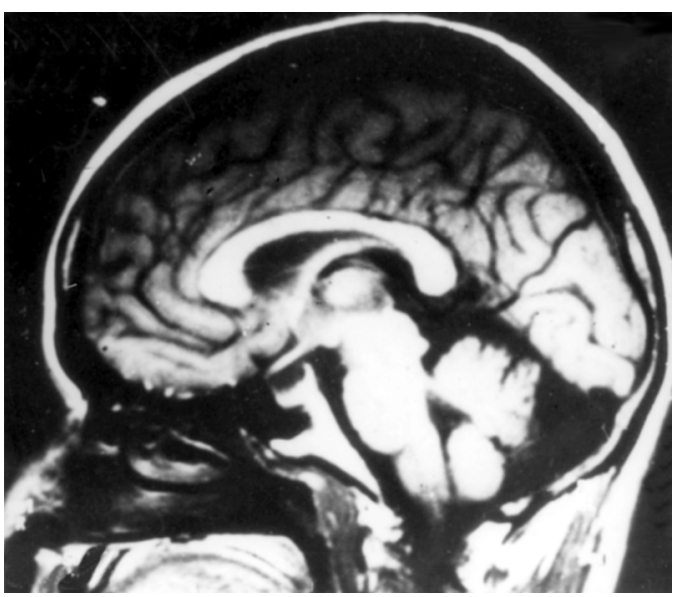

Figure 3 MRA after treatment

\section{Discussion}

APLS, first described in 1983, is an important prethrombotic disorder associated with a specific group of antibodies. ${ }^{1}$ It is more frequent in females. Hughes originally studied it in patients with systemic lupus erythematosus but found that the patients had "atypical lupus or no lupus at all", hence the concept of primary APLS. ${ }^{2}$ B2-Glycoprotein and other such proteins are required for the binding of antibodies to phospholipids. ${ }^{3}$ Treatment is with long-term anticoagulants and aspirin. ${ }^{4}$ It can be further classified into primary and secondary varieties.

In 1965, Ian Bruce Sneddon, a British dermatologist, had described patients with livedo reticularis and cerebrovascular accidents which he attributed to some form of vasculitis. ${ }^{5}$ Later, this entity was found to be associated with the presence of antiphospholipid antibodies (Sneddon's syndrome). ${ }^{6}$ Its mode of inheritance is autosomal dominant and only 126 cases have been reported in the literature up to 
1992. We would strongly advise investigation of a young individual with stroke and skin lesions for antiphospholipid antibodies.

\section{Final diagnosis}

Sneddon's syndrome.

Keywords: stroke; livedo reticularis; antiphospholipid syndrome; anticoagulants; Sneddon's syndrome

1 Hughes GRV. Thrombosis, abortion, cerebral disease and the lupus anticoagulant. BMF 1983;287:1088-9.

Hughes GRV. The anticardiolipin syndrome. Clin Exp Rheumatol 1985;3:285-6.

3 Roubey RAS. Autoantibodies to phospholipid binding plasma proteins:a new view of lupus anticoagulants and other antiphospholipid antibodies. Blood 1994;84:2854-67.

4 Khamashta MS, Cuadro MJ, Mujic F, et al. The management of thrombosis in antiphospholipid syndrome. N Engl F Med 1995;332:993.
5 Sneddon IB. Cerebrovascular lesions and livedo reticularis. Br f Dermatol 1965;77:180.

6 Rebollo M, Val JF, Garijo F, et al. Livedo reticularis and cerebrovascular lesions (Sneddon's syndrome). Brain 1983; 106:965-79.

\title{
A young man with a characteristic syndrome
}

\author{
J Ray, D O’Gradaigh, R Tighe
}

A 21-year-old man presented with a 12-day history of feverish symptoms with a sore throat, polyarthralgia, and left-sided pleuritic pain. On direct questioning, a recent history of nasal crusting and weight loss was elicited. His right shoulder was painful and the pain in his left hip made walking increasingly difficult. On examination, he was mildly icteric, thin, and looked unwell with a low-grade pyrexia and very tender cervical lymphadenopathy. There were petechiae and ulcerations on the palate. The left groin was also tender and a fluctuant swelling was palpable on the lateral border of the thigh $10 \mathrm{~cm}$ below the anterior superior iliac spine. Active and passive movement of the left hip in all directions was limited by pain, whilst a full range of movement was seen in the right shoulder.

Investigations revealed haemoglobin $10.2 \mathrm{~g} / \mathrm{dl}$, mean corpuscular volume $90.1 \mathrm{fl}$, platelets 310 $\times 10^{9} / 1$, white blood cell count $14.9 \times 10^{9} / 1$ (neutrophils 12.56 , lymphocytes 1.13 , eosinophils 0.01 ), C-reactive protein $157 \mathrm{mg} / \mathrm{l}$, erythrocyte sedimentation rate $67 \mathrm{~mm}$, bilirubin $58 \mathrm{~m} / \mathrm{h}$ $\mu \mathrm{mol} / 1, \gamma$-glutamyl transpeptidase $211 \mathrm{IU} / 1$, alanine transaminase $114 \mathrm{IU} / 1$, prothrombin time $17.3 \mathrm{~s}, \mathrm{XDP}<10 \mathrm{mg} / \mathrm{dl}$, cANCA weakly positive. Urine microscopy was normal, Paul Bunnel test for Epstein Barr virus was negative, and a ventilation/perfusion scan showed a low probability of pulmonary embolus. A transthoracic echo and X-ray of the left hip were normal. His chest X-ray is shown in the figure.

Norfolk and Norwich Hospital, Brunswick

Road, Norwich, Norfolk NR1 3FR, UK J Ray

D O'Gradaigh $\mathrm{R}$ Tighe

Submitted 22 January 1999 Accepted 3 June 1999

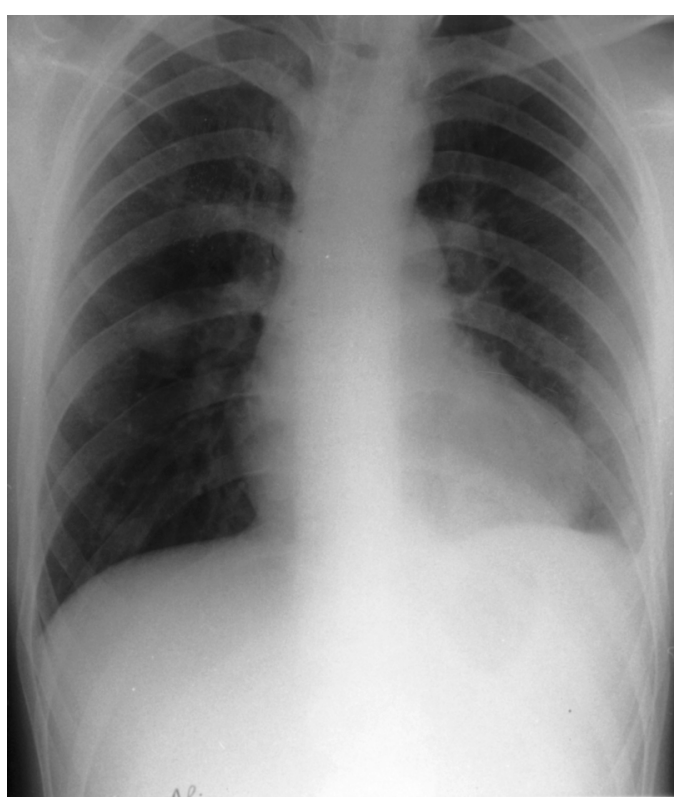

Questions

1 How would you report this chest X-ray?

2 What further investigations are required?

3 What is the most probable diagnosis? 


\section{Answers}

QUESTION 1

The chest X-ray demonstrates multiple lesions. On the left side is a cavitating lesion in the region of the heart shadow near the costovertebral angle.

QUESTION 2

Blood cultures and aspiration of the left hip using ultrasound guidance should be performed.

QUESTION 3

The most probable diagnosis is Lemierre's syndrome. $^{12}$

\section{Discussion}

The differential of multiple cavitating lung lesions is broad and includes infections, inflammatory conditions, and metastic disease. An infection with embolisation would be in keeping with the history and examination findings. A range of infective conditions would need consideration, for example, infective endocarditis, tuberculosis, or opportunistic infection in an immunocompromised individual.

Ultrasound of the left hip joint demonstrated an effusion which extended laterally and this was aspirated. Subsequent culture isolated Fusobacterium necrophorum. This Gramnegative anaerobe can cause a rare septicaemia which is also known as Lemierre's syndrome. The infection was successfully treated with a 2 -week course of intravenous antibiotics $1.2 \mathrm{~g}$ benzylpenicillin qid and $500 \mathrm{mg}$ metronidazole tid, in accordance with proven microbiology sensitivities), followed by 2 weeks of oral antibiotics.

A differential diagnosis of Wegener's granulomatosis ${ }^{3}$ was considered in view of the arthralgia, upper respiratory tract involvement and systemic upset. On immunofluorescence staining for ANCA, our patient had a weakly positive reaction with a cytoplasmic pattern. However, further clarification is important, and in this case, ELISA identified reactivity to bacterial permeability inhibitor, suggesting the antibodies were secondary to infection. A differential antigen, PR3, is strongly associated with Wegener's granulomatosis. Furthermore, the liver enzyme abnormalities and very short prodrome militate against this diagnosis, and while arthralgia can be marked, joint effusions are uncommon. Histological confirmation is essential before considering immunosuppression, and in this case, the microbiology from the hip effusion suggested the correct diagnosis, and obviating the need for further investigation. The consequences of undertaking immunosuppression in this patient, assuming a diagnosis of Wegener's granulomatosis, could have been very serious.

Lemierre's syndrome is caused by an acute oropharyngeal infection with secondary septic thrombophlebitis of the internal jugular vein, which is frequently complicated by metastatic infection. Painful cervical lymphadenopathy is usual. Systemic infection, characteristically involves the lung and joints, as occurred with our patient. Other sites of infection include liver, spleen, kidney and meninges. The early empirical use of antibiotics in the treatment of pharyngitis makes this a rarely seen condition. Lemierre's description of the clinical findings constituted, "a syndrome so characteristic that mistake is almost impossible."

\section{Final diagnosis}

Lemierre's syndrome (metastatic Fusobacterium necrophorum infection).

Keywords: Lemierre's syndrome; necrobacillosis
1 Golpe R, Marin B, Alonso M. Lemierre's syndrome (necrobacillosis). Postgrad Med f 1999;75:141-4.

2 Lemierre A On certain septicaemias due to anaerobic organisms. Lancet 1936;1:701-3.

3 Leavitt RY, Fauci AS, Bloch DA, et al. The American College of Rheumatology criteria for the classification of Wegener's granulomatosis. Arthritis Rheum 1990;33:1101-7.
4 Vohra A, Saiz E, Ratzan KR. A young woman with a sore throat, septicaemia, and respiratory failure. Lancet 1997 350:928. 


\title{
The collar sign
}

\author{
K B Agrawal, R T Kamble, P Sundaram, J M Joshi
}

A previously healthy 26-year-old man presented with the chief complaint of acute onset left-sided pleuritic type of chest pain. On enquiry he gave a history of blunt trauma to the lower chest and the abdomen due to a fall 6 years earlier which had been managed conservatively. On examination, the patient was obviously distressed and had tachycardia and tachypnoea. Breath sounds were absent in the left infrascapular and infra-axillary regions. His haematological and biochemical parameters were within normal limits. Arterial blood gases revealed hypoxaemia with $\mathrm{PaO}_{2} 66$ $\mathrm{mmHg}, \mathrm{PaCO}_{2} 36 \mathrm{mmHg}, \mathrm{pH}$ 7.41. Chest X-ray and computed tomography (CT) scans are shown in figures $1-3$.
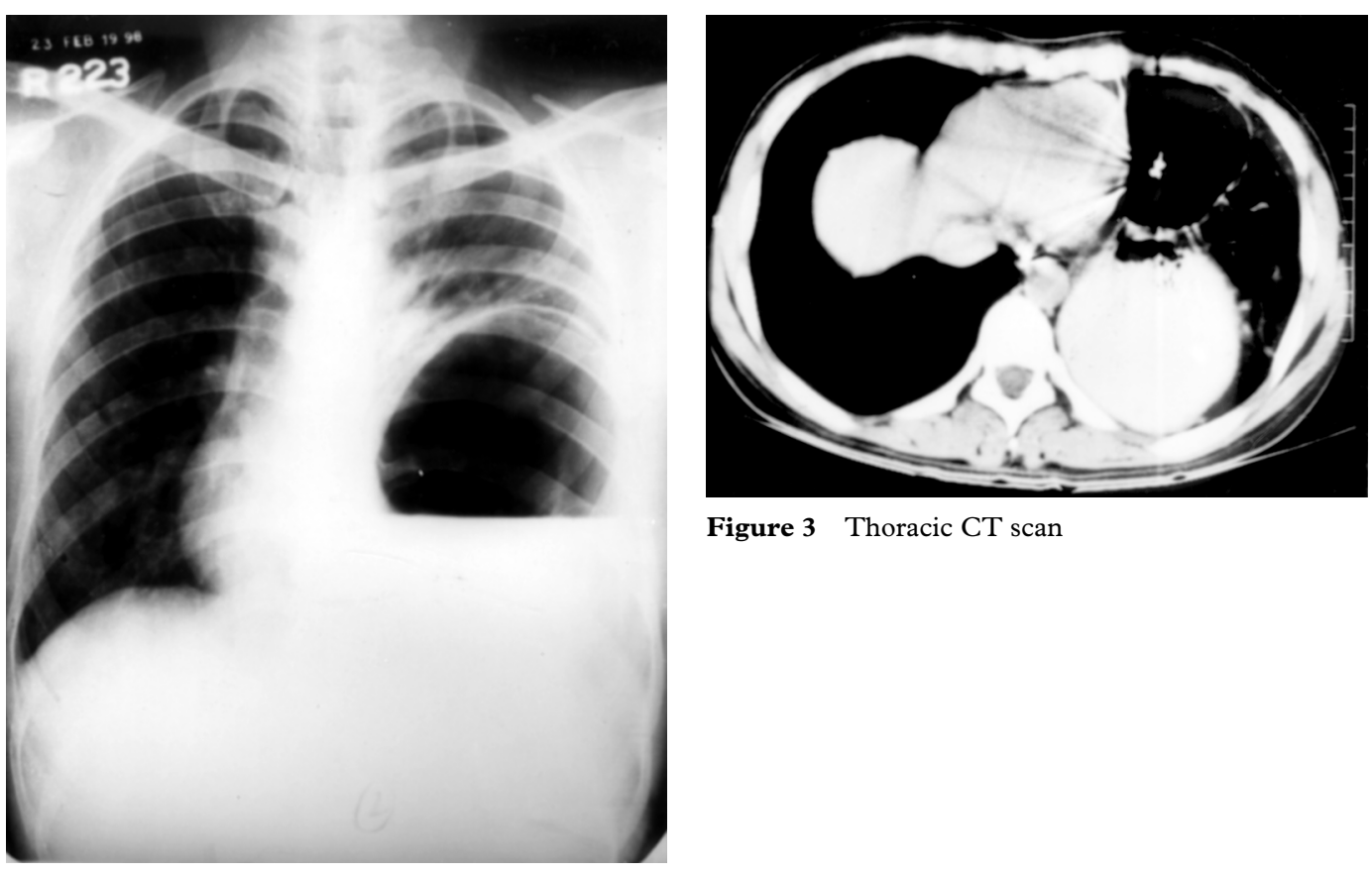

Figure 3 Thoracic CT scan

Figure 1 Chest X-ray

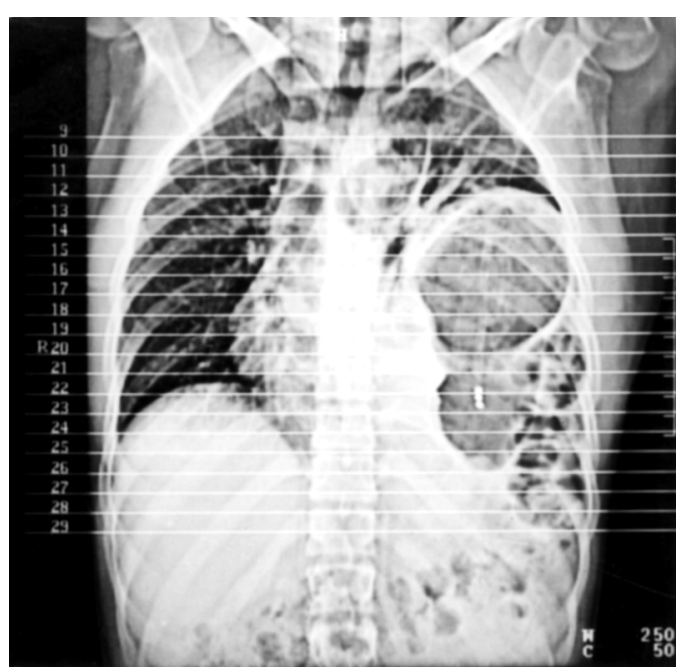

\section{Questions}

1 What is the differential diagnosis of the chest $\mathrm{X}$-ray?

2 What do the CT scans show?

Department of

Respiratory Medicine, BYL Nair Ch Hospital, Mumbai Central, Maharashtra, 400008 India

K B Agrawal

R T Kamble

P Sundaram

J M Joshi

Correspondence to Dr JM Joshi

Accepted 26 May 1999
Figure 2 Thoracic CT scan (scout scan) 


\section{Answers}

QUESTION 1

The chest X-ray shows an apparent elevation of the left hemidiaphragm with a large gas collection below it. The diagnostic considerations include eventration or paralysis of the diaphragm and diaphragmatic hernia.

QUESTION 2

On a chest X-ray it may be difficult to determine whether an abdominal organ lying unusually high is located beneath an elevated hemidiaphragm (in conditions like eventration or paralysis of hemidiaphragm) or is herniating through a deficit in the diaphragm. ${ }^{1}$ In this case a diagnosis of herniation can be easily made on the basis of the CT scans, which show focal constriction of the stomach at the site of herniation (collar sign). ${ }^{2}$

\section{Clinical course}

On the basis of the clinical and radiological findings, a diagnosis of traumatic diaphragmatic hernia was made. Surgery confirmed the presence of an $8-\mathrm{cm}$ tear in the posterolateral aspect of the left hemidiaphragm along with herniation of the stomach, transverse colon and spleen in the thorax. The patient made an uneventful recovery postoperatively.

\section{Discussion}

Diaphragmatic rupture occurs in approximately $5 \%$ of patients who have experienced major blunt trauma, ${ }^{3}$ and $90 \%$ of the diaphragmatic ruptures are on the left side. Left-sided hemidiaphragmatic injury predominates probably because of the protective effect of the liver on the right hemidiaphragm and/or underdiagnosis of right-sided injuries. The clinical diagnosis of laceration of the hemidiaphragm with herniated viscera is difficult, but may be suggested by bowel sounds auscultated in the lower thorax, unilateral absence of breath sounds, respiratory distress, and/or a scaphoid abdomen. Often, bedside physical findings are masked by concurrent abnormalities or these signs may be overlooked because of more apparent and life-threatening injuries in the acute post-trauma period. An early correct diagnosis is made in less than $50 \%$ of cases. Because the intrathoracic pressure is lower than the intra-abdominal pressure, there is progressive herniation of abdominal contents into the thorax. Carter et $a l^{4}$ have proposed three time phases following traumatic diaphragmatic rupture. The acute phase extends from the time of injury to 14 days later. If the patient survives the initial trauma and the hernia is not manifest within the first 14 days, the second or interval phase is entered. This interval phase extends until the third stage which is the phase of obstruction or strangulation. Delayed presentation of diaphragmatic rupture with visceral herniation and strangulation is associated with higher morbidity and mortality rates than when the correct diagnosis is made and the condition managed acutely.

\section{Learning points}

- a high index of suspicion should be maintained for the possibility of traumatic diaphragmatic hernia in all cases of blunt trauma

- CT enables detection of the majority of such diaphragmatic tears

- the most common findings include a localised defect of the diaphragm, the absent diaphragm sign and herniation of solid or hollow organs and omentum into the hemithorax

- the identification of the 'collar sign' on CT scan helps to differentiate diaphragmatic hernia from paralysis or eventration of the diaphragm

Chest X-ray is the principal screening method for thoracic injury after blunt trauma. Diagnostic or strongly suggestive findings on chest X-ray include the definite presence of air-filled viscera or the tip of the nasogastric tube above the diaphragm, as well as a diaphragm that is 'very elevated'. Obscuration of a non-elevated hemidiaphragm also suggests diaphragmatic hernia. The value of chest $\mathrm{X}$-rays in diagnosing right-sided traumatic diaphragmatic rupture is limited.

CT of the diaphragm has been reported to be useful in assessing for traumatic diaphragmatic rupture. ${ }^{5}$ One can usually see more of the left hemidiaphragm than the right. The posterolateral portions of both hemidiaphragms are usually best demonstrated, and thus the tears at those sites are more readily detected. The dome of the diaphragm is a difficult area to demonstrate with CT, the plane of the scan being tangential to the diaphragm at that point. The most common finding in diaphragmatic tear is a sharp discontinuity of the diaphragm. A large gap between the torn ends of the diaphragm results in the absent diaphragm sign. On the transverse CT scan, the abdominal contents lie central to the diaphragm and the thoracic contents lie peripherally. Intrathoracic herniation of peritoneal fat or organs can thus be identified by means of the abnormal position of these structures relative to the diaphragm. The structures that most commonly herniate through the defect are peritoneal fat, stomach, and colon. On transverse CT scans, to determine whether an abdominal organ lying in an unusually cephalad position, is located beneath an elevated hemidiaphragm or is herniating through a defect in the diaphragm, the presence of a focal constriction of the bowel or stomach at the site of herniation (collar sign) is very useful. The use of contrast material is essential in the demonstration of the collar sign in herniated stomach or colon when the diagnosis is made on the basis of findings in upper gastrointestinal or barium enema series. ${ }^{4}$

Other imaging methods that have been reported to be of value in evaluating the diaphragm include X-rays taken after nasogastric tube placement, fluoroscopy, upper and lower gastrointestinal contrast examination, sonography, magnetic resonance imaging, contrast or air peritoneography and liver/spleen scintigraphy. The appearance of herniated liver on the nuclear liver/spleen scan is rather 
characteristic and its use is recommended. Newer techniques such as spiral CT scanning allow better visualisation of the dome of the diaphragm owing to multiplanar capabilities.

Keywords: diaphragmatic hernia; chest trauma; collar sign
1 Probert NR, Havard C. Traumatic diaphragmatic hernia Thorax 1961;16:99-113.

2 Naidich DP, Zerhouni EA, Siegelmann SS. Computed tomog raphy and magnetic resonance of the thorax. New York, NY Raven, 1991.

3 Meyers BF, McCabe CJ. Traumatic diaphragmatic hernia: occult marker of serious injury. Ann Surg 1991;218:783-90.

\section{Final diagnosis}

Left-sided traumatic diaphragmatic hernia.
4 Carter BN, Giusefti J, Felson B. Traumatic diaphragmatic hernia. AfR 1951;65:56-71.

5 Worthy SA, Kang EY, Hartman JE, Kwong JS, Mayo JR, Muller NL. Diaphragmatic rupture: CT findings in 11 patients. Radiology 1995;194:885-8.

\title{
An adolescence girl with an anterior mediastinal mass
}

\author{
W S Chow, A W C Kung
}

A 14-year-old Chinese girl was referred to us for assessment of her thyrotoxicosis before corrective surgery of genu varus. On questioning, she mentioned significant weight loss with occasional palpitations. She had also experienced episodic lower limb proximal muscle weakness, but there was no history of paralysis.

Physical examination showed sinus tachycardia of 110 beats/min. There was no lid retraction or lid lag. Her goitre was enlarged three-fold with audible bruit, but there was no retrosternal extension. Eye examination revealed no evidence of Graves' ophthalmopathy. Abdominal examination was normal. There was no lymphadenopathy, splenomegaly nor pretibial myxoedema. Her proximal muscle power was grade $4 / 5$ and the jerks were all brisk.

Investigations showed a raised free thyroxine of $>170 \mathrm{pmol} / 1$ (normal range 10-19 pmol/1) and a suppressed thyrotropin of $<0.03 \mathrm{mIU} / 1(0.35-5.5 \mathrm{mIU} / \mathrm{l})$. Chest X-ray, however, revealed a mediastinal shadow with clear lung field. Complete blood count and routine biochemistry were normal. Acetylcholamine receptor antibody was normal. A computed tomography (CT) scan was arranged for further visualization of the mediastinal mass (figure 1). Carbimazole and propanolol were started for her thyrotoxicosis. Subsequently she became euthyroid and her muscle weakness disappeared. A follow-up CT scan, taken 8 months later, is shown in figure 2 .

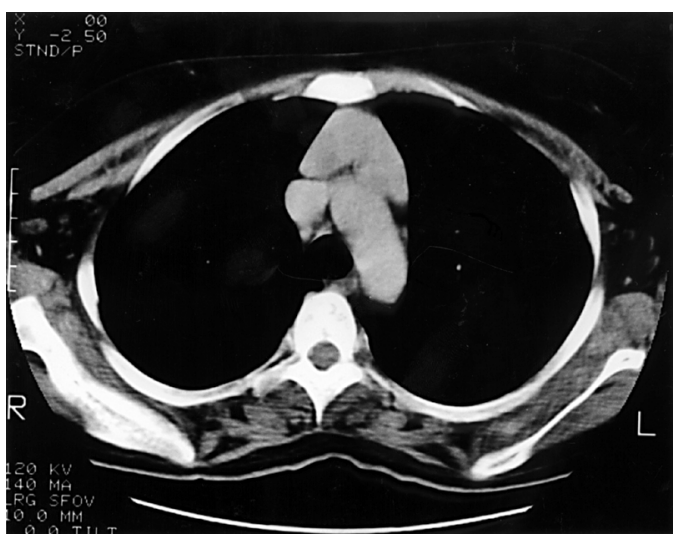

Figure 1 Initial CT scan

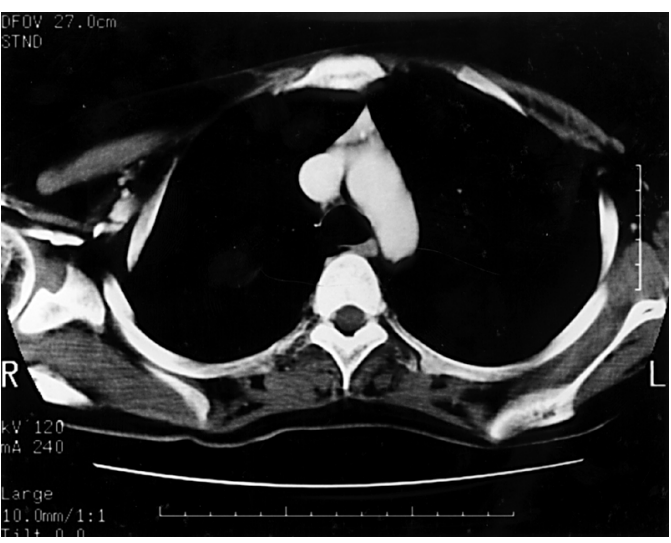

Figure 2 Follow-up CT scan 8 months later

\section{Questions}

1 What is the abnormality seen in the initial CT scan?

2 What are the differential diagnoses?

3 What is the underlying cause of the abnormality? 


\section{Answers}

QUESTION 1

The initial CT scan showed an anterior mediastinal mass, which is a diffusely enlarged thymus. Since the normal configuration of the gland was preserved, the findings were compatible with thymic hyperplasia. There was no abnormal mediastinal lymphadenopathy.

QUESTION 2

The differential diagnosis of an anterior mediastinal mass includes a variety of clinical entities (box).

It is useful to recognise the clinical association of thymic hyperplasia with hyperthyroidism, although massive enlargement of the thymus detected radiologically has only rarely been reported. Some studies have shown that approximately $38 \%$ of patients with thyrotoxicosis have an abnormal thymus gland, and biopsy shows the formation of medullary lymphoid follicles. ${ }^{1}$ Michie et al showed that the enlarged thymus regresses upon treatment of hyperthyroidism with antithyroid agents. ${ }^{2}$ The decrease in thymic size on treatment with antithyroid drugs could be related to a direct immunosuppressant effect of the antithyroid drugs or an indirect effect by lowering of circulating thyroid hormones. It has been reported that exogenous thyroxine treatment in animals could result in thymic enlargement. ${ }^{3}$

QUESTION 3

Recent studies have demonstrated mRNA and protein expression of the thyroid-stimulating hormone (TSH) receptor in thymic tissues of patients with Graves' disease. ${ }^{4}$ As Graves' disease results from the development of antibodies against the TSH receptor, the

1 Michie W, Gunn A. The thyroid, the thymus and autoimmunity. Br 7 Clin Pract 1966;20:9-13.

2 Michie W, Beck JS, Mahaffy RG, Honein EF, Fowler GB Quantitative radiological and histological studies of the thymus in thyroid disease. Lancet 1967;1:691-5.

3 Scheiff JM, Cordier AC, Haumont S. Epithelial cell proliferation in thymic hyperplasia induced by triiodothyronine. Clin Exp Immunol 1977;27:516-21.

\section{Differential diagnoses}

- thymic hyperplasia

- thymoma

- mediastinal lymphoma

- retrosternal goitre

- germ cell neoplasm

- thymic hyperplasia associated with Graves' disease

thymic TSH receptor may act as an autoantigen responsible for the initiation or perpetuation of the autoimmune response. However, the underlying pathophysiology of thymic hyperplasia is still not well delineated.

\section{Outcome}

Bergman et al recommended deferring invasive diagnostic manoeuvres until the effects of specific antithyroid treatment could be evaluated. ${ }^{5}$ We adopted this approach with close imaging monitoring. The thymus soon returned to normal size after euthyroidism had been achieved with antithyroid drugs. Although there was no histological confirmation, thymic hyperplasia is the most probable diagnosis based on the sequence of events.

\section{Final diagnosis}

Thymic hyperplasia.

Keywords: thymic hyperplasia

4 Murakami M, Hosoi Y, Negishi T, et al. Thymic hyperplasia in patients with Graves' disease. F Clin Invest 1996;98:2228

5 Bergman TA, Mariash CN, Oppenheimer JH. Anterior mediastinal mass in a patient with Graves' disease. 7 Clin Endocrinol Metab 1982;55:587-8. 


\title{
A pain in the neck
}

\author{
G V McDonnell, K E Bell, S A Hawkins
}

A previously healthy and independent 84-year-old woman was referred to the neurology department with leg weakness. Nine days earlier she had experienced an acute onset of severe neck pain whilst sitting at rest, which radiated down both arms. Although initially feeling unable to move, she attended a casualty department where analgesics and diazepam were prescribed and she was sent home. However, on leaving the hospital her legs felt heavy and she required the assistance of two people.

At home the next day she was unsteady on her feet and fell. Over the ensuing 4 days she developed leg cramps, urinary retention and constipation, necessitating her admission to hospital. On neurological examination, cranial nerves were intact but there was a quadriparesis mainly affecting the distal arm muscles and leg flexors with grade 3-4/5 power in the wrist extensors and finger abductors, $4 / 5$ power in quadriceps and $3 / 5$ strength in the hamstrings. Plantar responses were bilaterally extensor. Neck movements were markedly restricted by pain. Because of the nature of the history and the distribution of clinical signs, magnetic resonance imaging (MRI) of the spine was undertaken (figure).
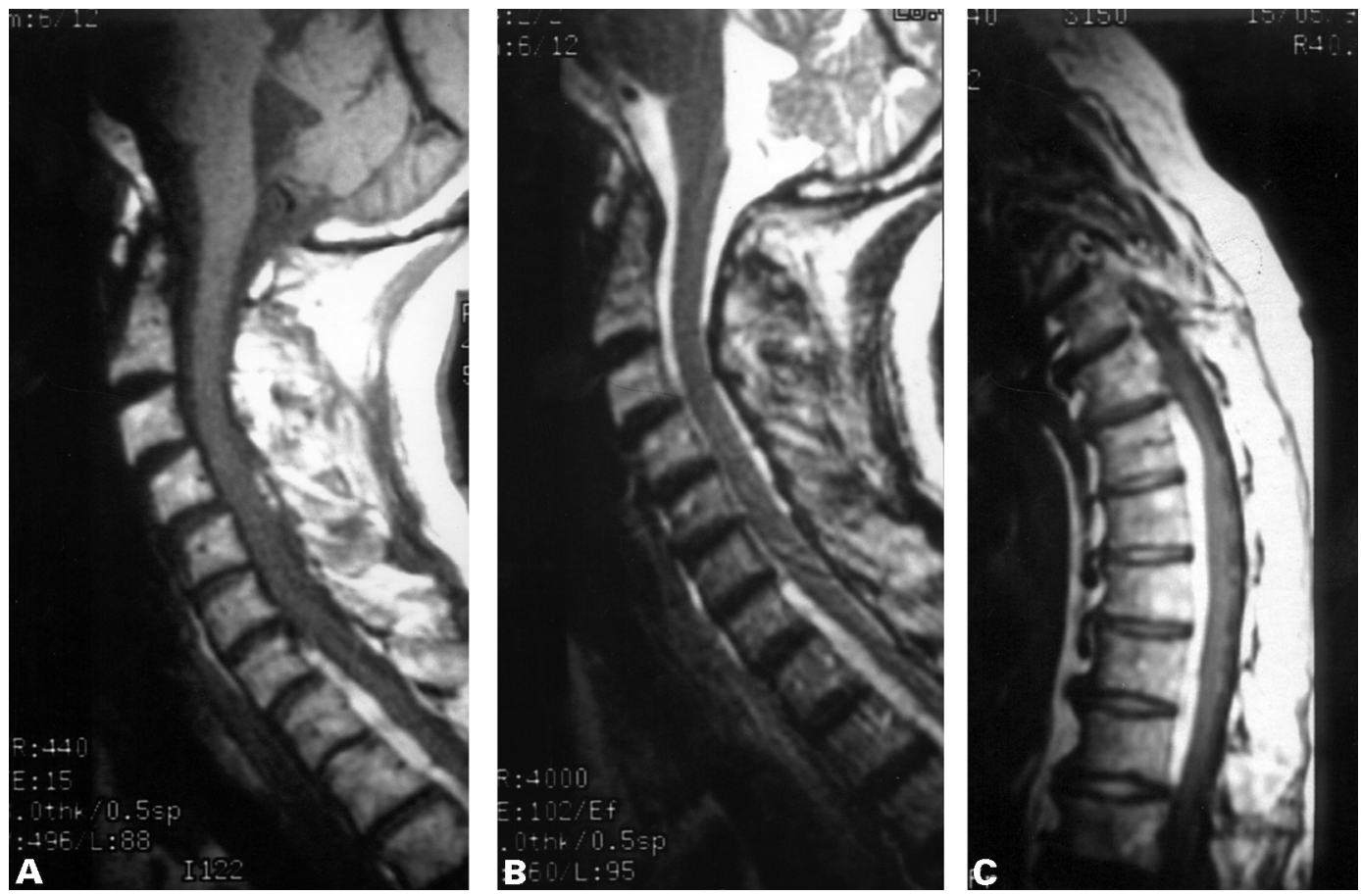

Northern Ireland
Regional Neurology

Service, Royal Victoria

Hospital, Belfast,

Northern Ireland

G V McDonnell

K E Bell

S A Hawkins

Correspondence to

Dr GV McDonnell,

Department of Neurology,

Ward 21, Quin House, Royal

Victoria Hospital, Grosvenor

Road, Belfast BT12 6BA,

Northern Ireland

Figure MRI images of the spine. (A) Sagittal T1-weighted image of the cervical spine (TR $440 \mathrm{~ms}$, TE $15 \mathrm{~ms}$ ), (B) sagittal T2-weighted image of the cervical spine (TR $4000 \mathrm{~ms}$, TE $102 \mathrm{~ms}$ ); (C) sagittal T1-weighted image of the dorsal spine (TR $440 \mathrm{~ms}$, TE $15 \mathrm{~ms}$ )

\section{Questions}

1 What is the differential diagnosis of the spastic quadriparesis in this patient?

2 What do the MRI scans show?

3 What is the diagnosis?

4 What further elements of the history are relevant and what other investigations are indicated?

Submitted 24 February 1999 Accepted 3 June 1999 


\section{Answers}

QUESTION 1

The differential diagnosis of spastic quadriparesis is obviously wide, as indicated by the non-exhaustive list in box 1 . However, the acute nature of the onset in this patient narrows the list considerably, with a vascular aetiology and an acute central cervical disc herniation being most likely, given the lack of any obvious spinal cord trauma. The absence of a prodromal illness or systemic upset weighs heavily against an inflammatory aetiology whilst demyelinating disease and hereditary spastic paraplegia would be unlikely to present for the first time in this late age group.

Although they would in any case have a more chronic course, syringomyelia would most commonly manifest with lower motor neurone signs in the arms and a dissociated sensory loss whereas motor neurone disease does not in general present with a 'pure' spastic quadriparesis. HTLV-1 mediated tropical spastic paraparesis usually spares the upper limbs and is rare in Caucasian populations.

\section{Differential diagnosis of spastic quadriparesis}

- vascular: anterior spinal artery occlusion, aortic dissection, arteriovenous malformation, epidural or subdural haematoma

- degenerative: central spondylosis, central cervical disc herniation, syringomyelia, Paget's disease

- neoplastic: intramedullary (glioma, ependymoma, lymphoma); extramedullary (neurofibroma, meningioma, ependymoma, bronchial and breast metastases, myeloma)

- inflammatory: viral, bacterial, fungal, parasitic, granulomatous, post-infectious, post-vaccine

- demyelinating: multiple sclerosis

- metabolic: subacute combined degeneration of the cord

- hereditary: hereditary spastic paraplegia

- other: motor neurone disease, HTLV-1 mediated tropical spastic paraparesis, parasagittal meningioma

Box 1

QUESTION 2

Figure (A) demonstrates high signal present anterior to the cord on T1-weighted images of the cervical spine from the level of C7. The T2-weighted image in figure (B) shows a similar area of high signal intensity arising from the same area. Figure (C) indicates that this area of high signal intensity also extends down the anterior surface of the dorsal cord and indeed reaches as far as the conus (not visible).

QUESTION 3

The MRI appearances are typical of a subacute haematoma in the epidural space. Within 24 hours of onset a haematoma is usually isointense with the cord on T1-weighted images but with the development of methaemoglobin in the haematoma it becomes of high signal intensity on both $\mathrm{T} 1-$ and $\mathrm{T} 2$-weighted images. The thin rim of low signal intensity just visible in the figures separating the haematoma from the cord is dura mater.

QUESTION 4

Although spinal epidural haematoma is 'spontaneous' with no obvious precipitating factors in over $50 \%$ of cases, certain conditions and triggers are believed to be associated. These include: bleeding diatheses associated with haematological disorders and with the use of anticoagulants and thrombolytics; spinal trauma including surgery and spinal puncture; tumours; hypertension, and arteriovenous malformations. Rarer associations have been reported with rheumatological conditions, alcohol, pertussis and pregnancy. Further questioning and investigations should therefore be directed appropriately. In this instance there had been no history of anticoagulant therapy, trauma or hypertension and there was no evidence of a tumour or vascular malformation on MRI. Clotting profile and platelet aggregation studies were normal.

QUESTION 5

The options in the management of this condition include surgical intervention, involving laminectomy and removal of the clot, or a conservative approach. In view of the extensive nature of the lesion and the advanced age of the patient, the latter approach was adopted. There was no further clinical deterioration and within 72 hours a slow clinical improvement was becoming apparent. Within 8 weeks there had been a full neurological recovery with return of sphincter function and the patient was living independently once more.

\section{Discussion}

Spinal epidural haematoma is an uncommon and rarely reported clinical entity which does, however, represent a neurological emergency. Although a painless onset has been described, this is exceptional and, as in this case, the disease is typically characterised by sudden onset of neck or back pain followed by sensory and motor dysfunction. The full symptom complex may evolve rapidly within one hour or may take weeks or even months to develop fully (box 2).

Early diagnosis may be difficult since a combination of interscapular and radicular pain can mimic an aortic dissection and radicular pain may be difficult to differentiate from pain due to a cardiac or pulmonary event. Acute

\begin{tabular}{|l|} 
Typical presentation of spinal epidural \\
haematoma
\end{tabular}

Box 2 
onset of pain in the upper neck may also raise suspicion of subarachnoid haemorrhage. However, bilateral symptoms of myelopathy in connection with neck or interscapular pain radiating into the upper extremities should lead to suspicion of cervical pathology including disc prolapse, spinal tumour or metastasis and epidural haematoma.

Spinal epidural haematomas occur in all age groups but are more common in middle or later life and are more frequent in males than females. Usually the haematoma lies in the dorsal epidural space but the anterior location demonstrated in this patient has been previously described. ${ }^{1}$ The most frequent levels of involvement are the cervical and thoracic segments with thoracolumbar and lumbar regions being less commonly affected. ${ }^{2}$ The extent of the lesion in this patient is unusual since most epidural lesions involve just 2-4 segments although a haematoma extending over 11 segments has been reported. ${ }^{3}$

Although no underlying cause for spinal epidural haematoma can be determined in at least $50 \%$ of cases, ${ }^{4}$ it is well recognised that predisposing factors include trauma, bleeding diatheses and anticoagulant therapy (box 3). Cases have also been reported as occurring in connection with apparently routine procedures such as epidural anaesthesia and diagnostic lumbar puncture. ${ }^{5}$ The significance of factors such as hypertension and underlying vascular malformations remains more controversial since it has been noted that hypertension is no more prevalent in patients with spinal epidural haematoma than in age-matched controls, ${ }^{4}$ and vascular malformations have been associated in less than $5 \%$ of cases at most. ${ }^{6}$

The MRI characteristics of spinal epidural haematoma are quite specific. ${ }^{7}$ On sagittal sections it is clearly outlined with tapering superior and inferior margins. Dura mater is seen as a curvilinear low signal separating the haematoma from the cord. Within 24 hours of onset the haematoma is isointense with the cord on T1-weighted images and heterogenous on T2weighted images. However, methaemoglobin accumulates after 24 hours causing $\mathrm{T} 1$ shortening and an increase in the signal on T1-weighted images. The differential diagnosis of such appearances should include spinal subdural haematoma, epidural neoplasm and abscess.

\section{Conditions associated with spinal epidural haematoma}

- bleeding diatheses

- drugs: aspirin; anticoagulants; thrombolytics

- spinal surgery, trauma, puncture

- spinal tumours

- arteriovenous malformations

- rheumatological disorders

- pregnancy

- alcohol

Box 3
The spinal subdural haematoma is far less frequent than the epidural haematoma and is rarely spontaneous, usually occurring in relation to anticoagulant therapy, blood dyscrasias or spinal trauma. MRI does not show a low signal dural rim. Epidural metastases give low signal or are isointense with the cord, with irregular margins and without tapering ends. These are generally associated with vertebral infiltration, which appears as diffuse or focal areas of homogenous low signal on T1weighted images. Epidural abscesses are fusiform and contiguous with an infected disc and vertebral bodies. They are centred on the disc and in most cases give lower signal than the spinal cord on MRI. Both metastases and abscesses usually also show enhancement on T1-weighted images post gadolinium.

In general, surgical intervention in cases of spinal epidural haematoma has been considered mandatory. Indeed it has been found that delay of more than 36 hours before decompressive laminectomy and evacuation of clot is associated with a poor prognosis. ${ }^{8}$ However in recent years there have been several reported instances of spontaneous recovery following conservative management in patients with mild or non-progressive neurological deficits. ${ }^{9}$ The outcome in this case lends further support to these observations.

In conclusion, although the differential diagnosis of spastic quadriparesis is wide, this case emphasises the importance of suspecting a diagnosis of spinal epidural haematoma in any patient presenting with acute onset of severe neck or back pain when this is associated with radicular symptoms or neurological signs consistent with cord compression. Predisposing factors should be sought and urgent surgical intervention should be considered although the potential for spontaneous recovery does exist in certain cases.

\section{Summary points}

- spinal epidural haematoma is a neurological emergency which occurs most commonly in middle life or later and is more common in males

- MRI is the diagnostic tool of choice

- cervical and upper thoracic spinal segments are most commonly involved

- about $50 \%$ of cases occur spontaneously

- bleeding/clotting disorders should be excluded

- urgent decompressive laminectomy and evacuation of clot is indicated in patients with significant or progressive deficits

- conservative management may be appropriate in mild and non-progressive cases and culminate in spontaneous recovery

Box 4

\section{Final diagnosis}

Spontaneous spinal epidural haematoma.

Keywords: epidural haematoma; MRI; spine 
1 Chen CJ, Fang W, Chen CM, Wan YL. Spontaneous spina epidural haematomas with repeated remission and relapse.

2 Rothfus WE, Chedid MK, Deeb ZL, Abla AA, Maroon JC, Sherman RL. MR imaging in the diagnosis of spontaneous epidural haematomas. F Comput Asst Tomogr 1987;11:851-4.

3 Larsson EM, Holtås S, Cronqvist S. Emergency magnetic resonance examination of patients with spinal cord symptoms. Acta Radiol 1988;26:69-75.

4 Groen RJM, Ponssen H. The spontaneous spinal epidural haematoma: a study of the aetiology. F Neurol Sci 1990;98:121-38.

5 Peltola J, Sumelhati M-L, Kumpulainen T, Dastidar P, Helén P. Spinal epidural haematoma complicating diagnostic lumbar puncture. Lancet 1996;347:131.
6 Foo D, Rosier AB. Preoperative neurological status in predicting surgical outcome of spinal epidural haematomas. Surg Neurol 1981;15:389-401.

7 Boukobza M, Guichard JP, Boissonet M, et al. Spinal epidural haematoma: report of 11 cases and review of the literature. Neuroradiology 1994:36:456-9.

8 McQuarrie IG. Recovery from paraplegia caused by spontaneous spinal epidural haematoma. Neurology 1978;28:2248.

9 Anderson TJ, Donaldson IMacG. Spontaneous resolution of cervical spinal epidural haematoma. Postgrad Med f 1989; 65:488-90.

\section{Epidural lipomatosis}

A 51-year-old man presented with radicularlike pain in the left lower limb and intermittent claudication lasting for 1 year. Pain in the legs was evoked by standing or walking more than 300 meters and rapidly relieved by sitting. Clinical examination only disclosed significant overweight (weight $100 \mathrm{~kg}$; height $163 \mathrm{~cm}$; BMI: $37.6 \mathrm{~kg} / \mathrm{m}^{2}$ ). Electromyography showed chronic neurogenic abnormalities in the muscles supplied by the left L5 nerve root. Magnetic resonance imaging (MRI) of the lumbar spine disclosed cauda equina compression by epidural lipomatosis (see figure on opposite page, A and B).

After 3 months on a hypocaloric diet leading to $20 \mathrm{~kg}$ weight loss, pain and claudication had completely resolved. MRI showed a dramatic regression of the epidural lipomatosis (figure, $\mathrm{C}$ and $\mathrm{D}$ ). In the differential diagnosis of lumbar stenosis, the clinician must keep in mind the possibility of epidural lipomatosis, even in patients without systemic glucocorticoid therapy or Cushing's disease.

YVES BOUTSEN

Department of Rheumatology

JULIAN DONCKIER

Department of Internal Medicine and Endocrinology UCL (Université Catholique de Louvain), University Hospital of Mont-Godinne, 5530 Yvoir, Belgium

Keywords: epidural lipomatosis

Submitted 3 February 1999

Accepted 12 July 1999 

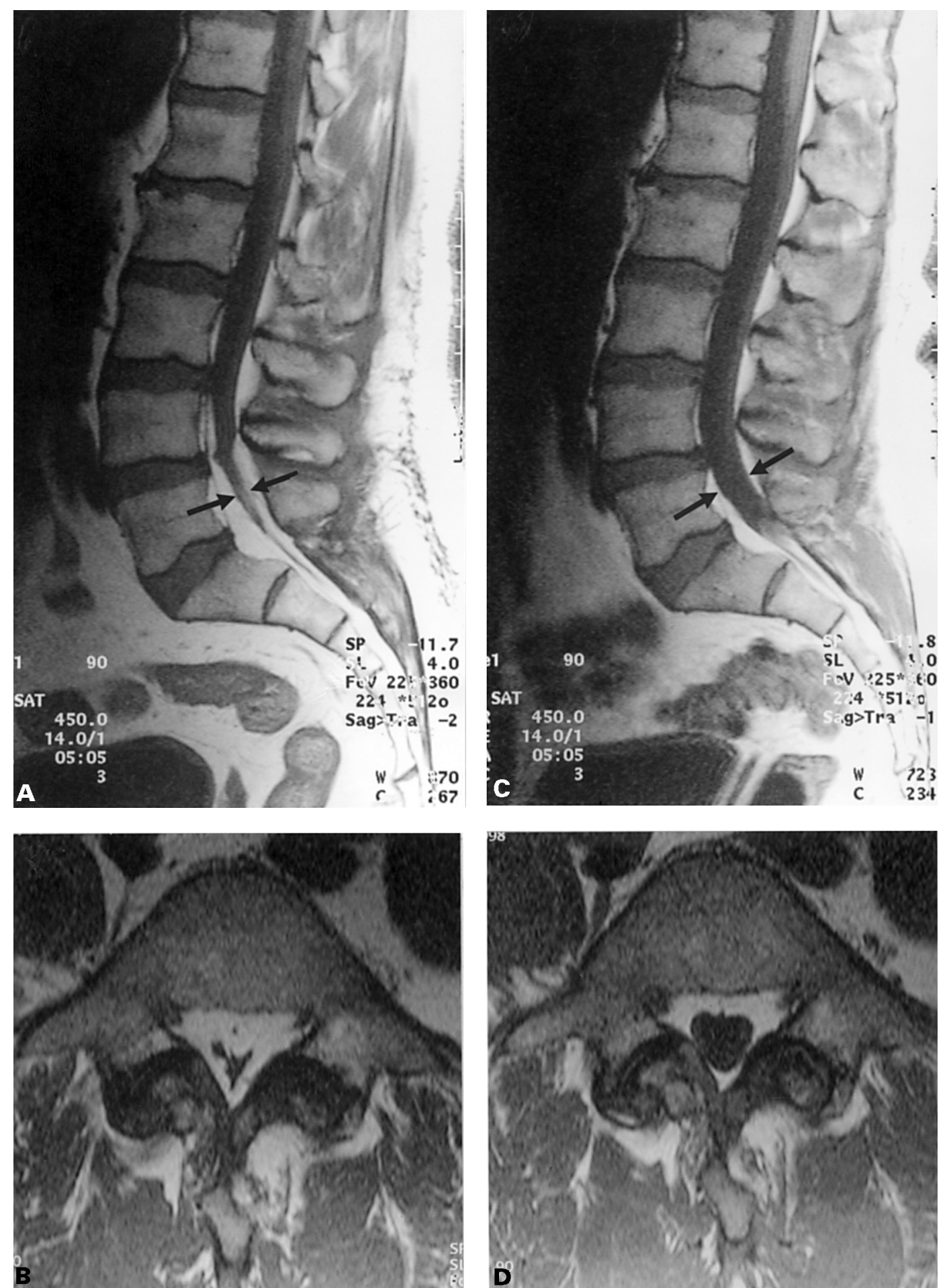

Figure Sagittal (A, C) and axial (B, D) T1-weighted MRI showing epidural fat-induced compression of the thecal sac before hypocaloric diet $(A, B)$ and normal apprearance after weight loss $(C, D)$ 